\title{
A Review of Immunomodulatory Effects of Fluoroquinolones
}

\author{
Shokrollah Assar (iD) ${ }^{\mathrm{a}, \mathrm{b}}$, Reza Nosratabadi (iD) ${ }^{\mathrm{C}}$, Hossein Khorramdel Azad (iD) ${ }^{\mathrm{d}, \mathrm{e}}$, \\ Javad Masoumie, Mahshad Mohamadif, and Gholamhossein Hassanshahi (iD) ${ }^{\text {d,e }}$
}

\begin{abstract}
almmunology of Infectious Diseases Research Center, Research Institute of Basic Medical Sciences, Rafsanjan University of Medical Sciences, Rafsanjan, Iran; 'Department of Microbiology, School of Medicine, Rafsanjan University of Medical Sciences, Rafsanjan, Iran; 'Department of Immunology, Faculty of Medicine, Kerman University of Medical Sciences, Kerman, Iran; ${ }^{d}$ Molecular Medicine Research Center, Research Institute of Basic Medical Sciences, Rafsanjan University of Medical Sciences, Rafsanjan, Iran; 'Department of Immunology, School of Medicine, Rafsanjan University of Medical Sciences, Rafsanjan, Iran; ${ }^{{ }^{f}}$ Medical Microbiology Research Center, Qazvin University of Medical Sciences, Qazvin, Iran
\end{abstract}

\begin{abstract}
Past researches indicate that some types of antibiotics, apart from their antimicrobial effects, have some other important effects which indirectly are exerted by modulating and regulating the immune system's mediators. Among the compounds with antimicrobial effects, fluoroquinolones (FQs) are known as synthetic antibiotics, which exhibit the property of decomposing of DNA and prevent bacterial growth by inactivating the enzymes involved in DNA twisting, including topoisomerase II (DNA gyrase) and IV. Interestingly, immune responses are indirectly modulated by FQs through suppressing pro-inflammatory cytokines, such as interleukin 1 (IL-1), IL-6, tumor necrosis factor-alpha (TNF-a), and super-inducing IL-2, which tend to increase both the growth and activity of T and B lymphocytes. In addition, they affect the development of immune responses by influencing of expression of other cytokines and mediators. This study aims to review past research on the immunomodulatory effects of FQs on the expression of cytokines, especially IL-2 and to discuss controversial investigations.
\end{abstract}

\section{KEYWORDS}

Fluoroquinolones; cytokine; anti-bacterial agents; immunomodulation; interleukin-2

\section{Introduction}

FQs are a broad range of synthetic antibiotics that are being widely consumed for their clinical features, including their availability, wide therapeutic index, and reasonable level of resistance (Mitscher 2005). These antimicrobial compounds exert their effects by entering into the cytosol of bacteria, binding to topoisomerase II (DNA gyrase) and IV, and inactivating these enzymes, especially in prokaryotes (Aldred et al. 2013). One of the distinctive features making FQs different from other natural antibiotics is their inherent physicochemical properties that help them to penetrate into the lipid bilayer of gramnegative bacteria to exert antimicrobial effects (Dalhoff and Schubert 2016; Idowu and Schweizer 2017). Given the broad range of antimicrobial activities, FQs are classified into four generations. The first generation includes oxolinic acid, inoxacin, flumequine, pefloxacin, rosoxacin, and nalidixic acid; the second generation encompasses ciprofloxacin, fleroxacin, lomefloxacin, nadifloxacin, norfloxacin, ofloxacin, and rufloxacin; the third

CONTACT Gholamhossein Hassanshahi ghassanshahi@gmail.com $\mathbf{B}$ Molecular Medicine Research Center, Research Institute of Basic Medical Sciences, Rafsanjan University of Medical Sciences, Rafsanjan, Iran 